\title{
Electrical Transport and Magnetic Properties of $\mathrm{La}_{2 / 3} \mathrm{~Pb}_{1 / 3} \mathrm{MnO}_{3}$ Thin Films
}

\author{
M. Baran, M. Berkowski, J. Fink-Finowicki,
}

S.J. LeWANDOWSKI, H. SZYMCZAK, R. SZYMCZAK

Institute of Physics, Polish Academy of Sciences

al. Lotników 32/46, 02-668 Warsaw, Poland

\author{
S.I. KhartcheV and Yu.V. MedvedeV \\ Physico-Technical Institute, National Academy of Sciences, Donetsk, Ukraine
}

The magnetic and transport properties of epitaxial $\mathrm{La}_{2 / 3} \mathrm{~Pb}_{1 / 3} \mathrm{CuO}_{3}$ thin films deposited on $\mathrm{SrLaGaO}_{4}$ substrate using dc magnetron sputtering technique are reported. The giant magnetoresistance effect (of about 50\% at magnetic field of $1 \mathrm{~T}$ ) was observed near the Curie temperature. Several mechanisms responsible for temperature dependence of resistivity are discussed. The effect of annealing was studied. It shifted the Curie temperature to the lower value, probably, because of the loss of oxygen.

PACS numbers: $75.30 .-\mathrm{m}, 72.15 . \mathrm{Gd}, 75.70 . \mathrm{Pa}$

\section{Introduction}

The $\mathrm{R}_{2 / 3} \mathrm{~A}_{1 / 3} \mathrm{MnO}_{3}(\mathrm{R}=$ rare earth, $\mathrm{A}=$ alkaline earth or lead $)$ compounds often exhibit a large magnetoresistance associated with a metal-insulator transition, which can take place at temperatures from about $30 \mathrm{~K}$ to above than $300 \mathrm{~K}$ [1-5]. Interest in the perovskite manganites has considerably increased since their fabrication as epitaxial thin films [6]. Until recently, much of the experimental work on the manganites has been devoted to the compounds $\mathrm{La}_{2 / 3} \mathrm{~A}_{1 / 3} \mathrm{MnO}_{3}$ with $\mathrm{A}$ being alkaline earth, while the properties of epitaxial $\mathrm{La}_{2 / 3} \mathrm{~Pb}_{1 / 3} \mathrm{MnO}_{3}$ films have not yet been reported. At the same time it has to be mentioned that the giant magnetoresistance was observed a long time ago [7] in $\mathrm{La}_{1-x} \mathrm{~Pb}_{x} \mathrm{MnO}_{3}$ single 
crystals. This material is unique among the manganites since for $0.26<x<0.4$ the Curie temperature is above $320 \mathrm{~K}$. Recently, it has been shown [8-10] using magnetic resonance technique that the magnetic mixed-phase state takes place in $\mathrm{La}_{0.7} \mathrm{~Pb}_{0.3} \mathrm{MnO}_{3}$ single crystals in the vicinity of $T_{\mathrm{c}}$.

\section{Experimental}

We present in this paper the measurements of electric transport and magnetic properties of epitaxial $\mathrm{La}_{2 / 3} \mathrm{~Pb}_{1 / 3} \mathrm{MnO}_{3}$ thin $(\approx 2000 \AA)$ films. The films were grown in 15 mTorr argon-oxygen mixture (1:1) by dc magnetron sputtering at deposition rate of $\approx 0.1 \mathrm{~nm} / \mathrm{s}$, plasma current of $100 \mathrm{~mA}$, and substrate-to-target distance of $7 \mathrm{~cm}$. The target of nominal stoichiometry was fabricated by reaction of high purity component oxides at $900^{\circ} \mathrm{C}(36 \mathrm{~h})$ followed by grinding, pressing into disk form $\left(40 \times 1 \mathrm{~mm}^{2}\right)$ and sintering at $1150^{\circ} \mathrm{C}(10 \mathrm{~h})$. X-ray diffraction measurements indicate a single-phase orthorombically distorted perovskite structure of the films. The films were grown on (001)-oriented $\mathrm{SrLaGaO}_{4}$ substrates at a temperature $T_{\text {sub }}=675^{\circ} \mathrm{C}$. After a deposition the films were cooled to room temperature in oxygen ( 250 Torr) at the rate of $5 \mathrm{~K} / \mathrm{min}$. Next, one of the studied films was annealed at $800^{\circ} \mathrm{C}$ for $3 \mathrm{~h}$ in oxygen flow. The crystal orientation of the studied films was determined by $\mathrm{X}$-ray diffraction measurements. The films were found to be (100)-oriented and to exhibit epitaxial in-plane alignment.

The electrical resistance of the samples was measured as a function of temperature and magnetic field using a standard four-probe dc technique. The measurements were performed with the external field applied parallel to the current direction. Magnetization was measured in a magnetic field up to $5 \mathrm{~T}$ over a temperature range of 5-300 K using a SQUID magnetometer (MPMS-5, Quantum Design).

\section{Results and discussion}

The temperature dependences of magnetization $M(T)$ in an applied field of $0.1 \mathrm{~T}$, for the as deposited and annealed samples, are shown in Fig. 1. The ferromagnetic Curie temperature $T_{\mathrm{c}}$ was determined by linear extrapolation of a maximal slope of the $M(T)$ data to zero. Using this procedure we obtained $T_{\mathrm{c}}=211 \mathrm{~K}$ for the as deposited film and $T_{\mathrm{c}}=176 \mathrm{~K}$ for the annealed film. The obtained values of $T_{\mathrm{c}}$ are considerably smaller than $T_{\mathrm{c}}=355 \mathrm{~K}$ reported for $\mathrm{La}_{0.7} \mathrm{~Pb}_{0.3} \mathrm{MnO}_{3}$ single crystal [7]. The shift of $T_{\mathrm{c}}$ to lower temperatures after annealing is also quite unexpected. It is in contrast to what is observed for $\mathrm{La}_{2 / 3} \mathrm{Ca}_{1 / 3} \mathrm{MnO}_{3}$ films deposited on $\mathrm{LaAlO}_{3}$ [11]. A possible explanation of both effects should, probably, be related to the oxygen deficiency. Similar effect was observed in $\mathrm{La}_{0.6} \mathrm{~Pb}_{0.4} \mathrm{MnO}_{3-\delta}$ thin films [12].

The temperature dependences of the resistivity $\rho(T)$ both for the as deposited and annealed films are shown in Fig. 2. The maximum of resistivity occurs 


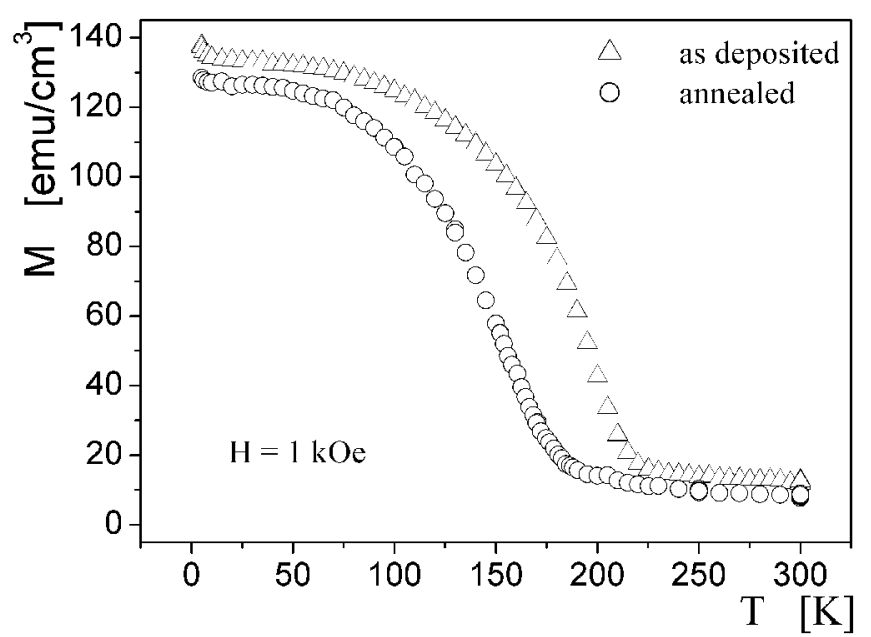

Fig. 1. Magnetization vs. temperature for as deposited $(\Delta)$ and annealed (o) films.

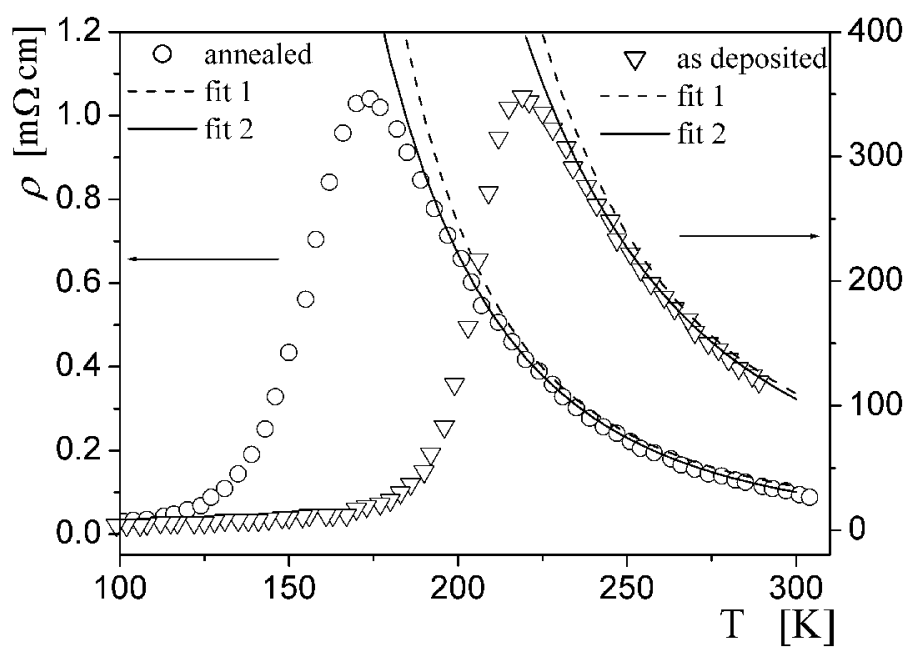

Fig. 2. Resistivity vs. temperature for as deposited $(\nabla)$ and annealed (o) films. The lines present least squares fits: continuous - small polaron hopping model, dashed semiconductor model.

very close to $T_{c}$ determined from the magnetization measurements. One can see that the drop in $\rho$ below $T_{c}$ is quite sharp, while above $T_{c}$ it is much less pronounced. This is caused by different mechanisms being responsible for the scattering processes in the temperature ranges below and above $T_{c}$. We have considered here two transport mechanisms for $T>T_{c}$ : (i) semiconductor-like mechanism [13] (see fit 1 in Fig. 2) and (ii) hopping of small polarons in adiabatic regime (see fit 2 in Fig. 2). The resistivity in the frame of hopping of small polaron model in 
adiabatic regime is described by

$$
\rho(T)=T \exp \left(\frac{E_{\mathrm{a}}}{k T}\right)
$$

where $E_{\mathrm{a}}$ is the activation energy. The activation energy determined as parameter of the fitting procedure was found to be $113 \mathrm{meV}$ and $100 \mathrm{meV}$ for as deposited and annealed film, respectively.

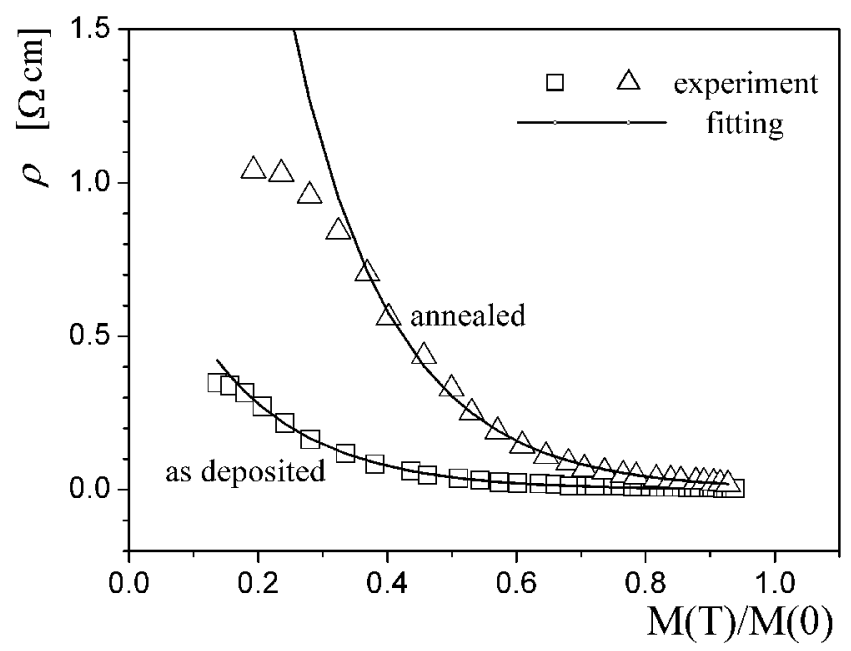

Fig. 3. Correlation between resistivity and magnetization for as deposited ( $\square$ ) and annealed $(\Delta)$ films. The lines present fits of Eq. (3) to experimental data. Fitting parameters $\rho_{\mathrm{m}}=1013$ and $7756, K=6.43$ and 6.48 for the as deposited and annealed films, respectively.

For the ferromagnetic region $\left(T<T_{c}\right)$ we have confirmed the existence of an interesting correlation between magnetization $M(T)$ and $\rho(T)$, which was observed in $\mathrm{La}_{0.7} \mathrm{Ca}_{0.3} \mathrm{MnO}_{3}$ thin films [14], and can be expressed as

$$
\rho(T)=\rho_{\mathrm{m}} \exp [-M(T) / W],
$$

where $W$ and $\rho_{\mathrm{m}}$ are fitting parameters. It is suggested in Ref. [14] that the transport below $T_{\mathrm{c}}$ described by Eq. (2) involves quasiparticle hopping. By transforming Eq. (2) to

$$
\rho(T)=\rho_{\mathrm{m}} \exp \left[-K \frac{M(T)}{M(0)}\right]
$$

it can be shown (see Fig. 3) that Eq. (3) describes correctly experimental results both for the as deposited and annealed films with practically the same values of parameter $K$ (6.43 and 6.48 , respectively).

The temperature dependences of the magnetoresistance effect $\left(\rho_{0}-\rho_{1 T}\right) / \rho_{0}$, in the magnetic field of $1 \mathrm{~T}$, are shown in Fig. 4, both for the as deposited and annealed films. 


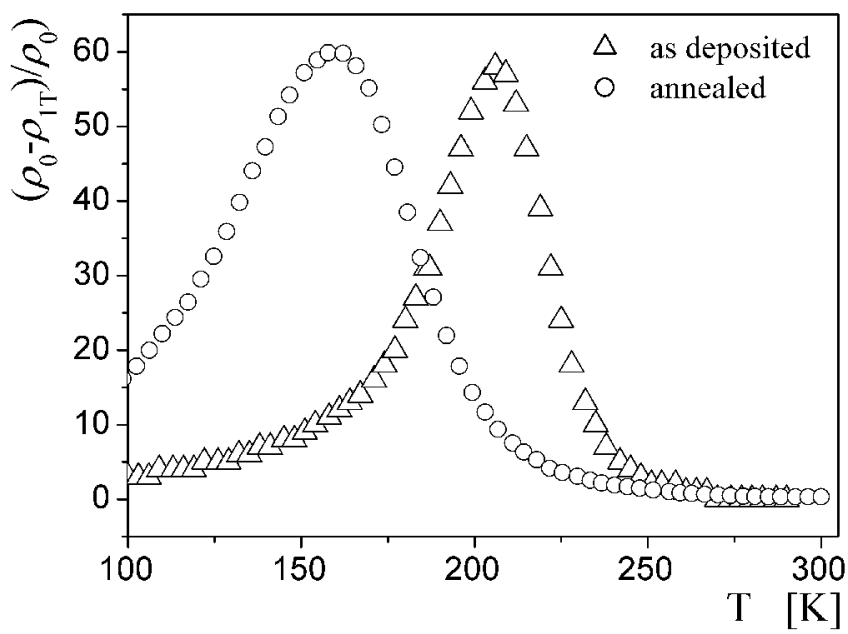

Fig. 4. Magnetoresistance effect vs. temperature for both studied films.

The results presented in this paper confirm the important role of quasiparticles in the determination of magnetic and transport properties of the investigated films.

To summarize, we have found giant magnetoresistance effect in $\mathrm{La}_{2 / 3} \mathrm{~Pb}_{1 / 3} \mathrm{MnO}_{3}$ films deposited on $\mathrm{SrLaGaO}_{4}$ substrate. We suggest that the mechanism responsible for this effect is related to the different roles played by quasiparticles below and above the Curie temperature.

\section{Acknowledgment}

The work was supported in part by the State Committee for Scientific Research (Poland) under contract no. PBZ/KBN/13/T08/99, and also in part within European Community program ICA1-CT-2000-70018 (Centre of Excellence CELDIS). Technical assistance of Yu. Bukhantsev and B. Kundys is also acknowledged.

\section{References}

[1] R. von Helmolt, J. Wecker, B. Holzapfer, L. Schultz, K. Samwer, Phys. Rev. Lett. 71, 2331 (1993).

[2] M. McCormack, S. Jin, T.H. Tiefel, R.M. Fleming, J.M. Phillip, R. Ramesh, Appl. Phys. Lett. 644, 3045 (1994).

[3] S. Jin, T.H. Tiefel, M. McCormack, R.A. Fastnacht, R. Ramesh, L. Chen, Science 264, 113 (1994).

[4] S. Jin, M. McCormack, T.H. Tiefel, R. Ramesh, J. Appl. Phys. 76, 6929 (1994). 
[5] J.Z. Sun, L. Krusin-Elbaum, S.S.P. Parkin, G. Xiao, Appl. Phys. Lett. 67, 2726 (1995).

[6] H.L. Ju, C. Kwon, Q. Li, L. Greene, T. Venkatesan, Appl. Phys. Lett. 65, 2108 (1994).

[7] C.W. Seale, S.T. Wang, Can. J. Phys. 48, 2024 (1970).

[8] N.V. Volkov, G.A. Petrakovski, V.N. Vasilev, K.A. Sablina, Fiz. Tverd. Tela 44, 1290 (2002).

[9] N.V. Volkov, G.A. Petrakovski, K. Sablina, K. Patrin, Acta Phys. Pol. A 105, (2004).

[10] N.V. Volkov, G.A. Petrakovski, V.N. Vasilev, K.A. Sablina, K.G. Patrin, J. Magn. Magn. Mater. 258-259, 302 (2003).

[11] D.C. Worledge, G.J. Snyder, M.R. Beasley, T.H. Geballe, J. Appl. Phys. 80, 5158 (1996)

[12] K.M. Satyalaksmi, S. Sundar Manoharan, M.S. Hedge, V. Prasad, S.V. Subramanyam, J. Appl. Phys. 78, 6861 (1995).

[13] G.J. Snyder, R. Hiskes, S. DiCarolis, M.R. Beasley, T.H. Geballe, Phys. Rev. B 53, 14434 (1996).

[14] M.F. Hundley, M. Hawley, R.H. Heffner, Q.X. Jia, J.J. Neumeier, J. Tesmer, J.D. Thompson, X.D. Wu, Appl. Phys. Lett. 67, 860 (1995). 